\title{
The decay constant of the first excited pion from lattice QCD
}

\author{
UKQCD Collaboration \\ C. McNeile \\ Department of Physics and Astronomy, The Kelvin Building \\ University of Glasgow, Glasgow G12 8QQ, U.K. \\ and \\ C. Michael \\ Theoretical Physics Division, Dept. of Mathematical Sciences, \\ University of Liverpool, Liverpool L69 3BX, UK
}

\begin{abstract}
We review the theory that predicts that the decay constant of the excited light pseudo-scalar mesons are suppressed in the chiral limit relative to the pion decay constant. We compute the decay constant of the first excited pion $\left(\pi^{\prime}\right)$ using unquenched QCD at a fixed lattice spacing with sea quarks of mass as low as a third the mass of the strange quark. The final result is very sensitive to the improvement conditions. We obtain $f_{\pi^{\prime}} / f_{\pi}=0.078(93)$ in the chiral limit.
\end{abstract}

\section{Introduction}

Holl et al. 1] have argued from Schwinger-Dyson equations and on general grounds that the decay constants of the excited light $0^{-+}$mesons should be suppressed relative to the pion decay constant. The decay constant of the first excited pion has also been found to be small in [2, 3, 4, 5, 6, 7, 8, 9]. In table 1we summarise the values for the decay constant of the first excited pion from various models. The predictions from these models for $f_{\pi^{\prime}}$ are indeed remarkably small. When we first heard about the result that the decay constant of the excited light pseudo-scalar could be as small a tenth of the pion decay constant, our first reaction was a combination of "that is remarkable" and "unbelievable". In this letter we check these predictions using lattice QCD.

Experimentally, the second lightest meson with $0^{-+}$quantum numbers, made out of light quarks, is the $\pi(1300)$. In our lattice calculation we use the notation $\pi^{\prime}$ for the first excited light pseudo-scalar meson.

From a (naive) quark model perspective it is surprising for the $\pi^{\prime}$ to have a highly suppressed decay constant. We define highly suppressed to be that the decay constant is less than $10 \%$ of the pion decay constant. In the naive quark model the leptonic decay constant for S-wave states is proportional to 
the wave-function at the origin. The wave function of the excited $0^{-+}$state would be smaller than the ground state, because the wave-function is more spread out than for the ground state. However, there is no obvious reason for a dramatic suppression of the decay constant. It is instructive to consider the decay constants of mesons with heavy quarks. Lakhina and Swanson [10 have recently computed the leptonic decay constant of mesons in the charmonium and bottonium systems and compared their values against experiment and lattice gauge theory. For example, Lakhina and Swanson [10] get good agreement from their model and the experimental results for the leptonic decay constants of the $J / \psi(411 \pm 7 \mathrm{MeV})$ and $\psi^{\prime}(279 \pm 8 \mathrm{MeV})$ mesons. The same decay constants have also been computed by Dudek et al. 11] from quenched lattice QCD. This type of modest suppression of the value for the decay constant of the excited meson relative to the decay constant of the ground state agrees with our (perhaps simple minded) intuition

Below is "simplistic" theoretical argument for the suppression of the decay constant of excited light pseudo-scalars Consider the axial current applied to the meson $X$ (here applied to mesons with $J^{P C}=0^{-+}$).

The decay constant $\left(f_{X}\right)$ of the state $X$ is defined by

$$
\left\langle 0\left|A_{\mu}\right| X\right\rangle=i f_{X} p_{\mu}
$$

where $A_{\mu}$ is the axial current. The partial conservation of the axial current 25] is

$$
\partial_{\mu} A_{\mu}=m_{q} \pi
$$

where $\pi$ is the interpolating operator for pion states (pseudo-scalar density) and $m_{q}$ is the quark mass. Equation 2 is an operator relation, hence is true between any states. This allows us to write:

$$
f_{X}=\frac{1}{m_{X}^{2}} m_{q}\langle 0|\pi| X\rangle
$$

For the lightest pseudo-scalar

$$
m_{X}^{2} \propto m_{q}
$$

so $f_{\pi}$ is non-zero in the chiral limit. For a meson that is not a Goldstone state, $m_{X}$ is non-zero in the chiral limit, hence $f_{X}$ will vanish in the chiral limit.

In the past, corrections to equation 2 from excited pion states 2, 12] have been considered for corrections to the Goldberger-Trieman relation. For example 2] introduced extended PCAC

$$
\partial^{\mu} A_{\mu}=\sum_{n} m_{\pi_{n}}^{2} f_{\pi_{n}} \pi_{n}
$$

where $\pi_{n}$ is the interpolating operator for the $\mathrm{n}$-th excited light $0^{-+}$meson.

The PDG 13] quotes the mass of the $\pi(1300)$ as $1300 \pm 100 \mathrm{MeV}$ with a decay width of between 200 to $600 \mathrm{MeV}$. The predominant decay mode is to $\pi \pi \pi$ (this includes $\rho \pi$ ). There is readable discussion about the experimental 


\begin{tabular}{|c|c|}
\hline Group & $f_{\pi^{\prime}} \mathrm{MeV}$ \\
\hline Volkov and Weiss [4] & 0.68 \\
Elias et al. [5] & $4.2 \pm 2.4$ \\
Maltman and Kambor [7] & $3.11 \pm 0.65$ \\
Andrianov et al. [6] & $0.52-2.26$ \\
Kataev et al. [19] [20] & 4.3 \\
\hline
\end{tabular}

Table 1: Summary of the values of the $\pi^{\prime}$ decay constant determined from models and sum rules. Our normalisation convention is $f_{\pi}=131 \mathrm{MeV}$.

issues with the $\pi(1300)$ state in [14]. Diehl and Hiller [8] estimate the leptonic decay constant of the $\pi(1300)$ to be less than $8.4 \mathrm{MeV}$ from the experimental bounds on the decay of the $\tau$ to $\pi(1300)+\nu_{\tau}$ from CLEO [13, 15].

The mass of the $\pi(1300)$ meson has been studied before using lattice QCD. Some quenched studies show reasonable agreement with experiment 16, 17. The $\pi(1300)$ decays via the strong interaction, so quenched calculations would not be expected to be so accurate for this state. Using the maximum entropy method the CP-PACS collaboration obtained the mass of the first excited pion to be $660(590) \mathrm{MeV}$ in the continuum limit of quenched QCD [17. The CPPACS collaboration [17 also computed the leptonic decay constant for the first excited $\rho$ meson. The ratio of the leptonic decay constant of the excited to ground rho meson, obtained by CP-PACS [17] was 0.41 . This is modest in accord with our quark model intuition. The unquenched lattice QCD calculation by the MILC collaboration, using improved staggered quarks, obtained the first excited pseudo-scalar to be at the mass $1362(41)(205) \mathrm{MeV}[18$.

\section{The lattice QCD calculation}

In 21] the UKQCD collaboration reported results for the pion decay constant from a two flavour unquenched calculation. The masses of the first excited light pseudo-scalar meson were reported but not the respective decay constants. The paper by UKQCD used a variational technique, where a matrix of correlators is fitted to a transfer matrix based model. This is one of the more reliable techniques to study excited states on the lattice. Variational and other lattice methods to extract properties of excited hadrons are reviewed in [22].

In this paper we report the decay constant of the first excited light pseudoscalar meson. The calculation used two flavours of degenerate sea quarks. The non-perturbatively improved clover action was used for the quarks with the Wilson gauge action. The lattice volume was $16^{3} 32$ and $\beta=5.2$. The lattice spacing is roughly $0.1 \mathrm{fm}$. The data for the pion decay constant and the mass of the $\pi^{\prime}$ state have already been published in [21, 23], so we do not repeat that here. Note that UKQCD also showed good agreement between the pion decay constant computed by UKQCD and the JLQCD collaborations 24.

One consequence of the suppression of the decay constants of the excited 


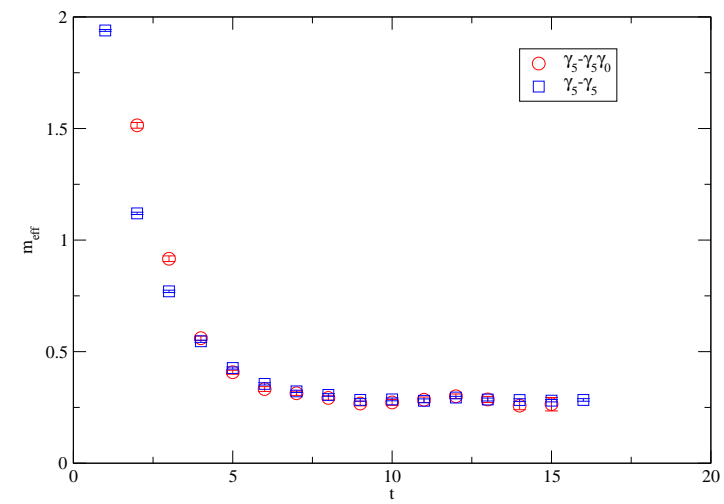

Figure 1: Effective mass for the $\gamma_{5}$ to $\gamma_{5}$ and for $\gamma_{0} \gamma_{5}$ to $\gamma_{5}$ correlators for $\kappa=$ 0.1355 .

pions would be that the correlators for $\gamma_{0} \gamma_{5}$ to $\gamma_{5}$ should show less excited contamination than for the $\gamma_{5}$ to $\gamma_{5}$ correlator. In figure 1 the effective mass plots for the two local correlators look very similar. Recall, that the flatter the effective mass plot, the less excited state contamination there is.

We use variational smearing to extract the required matrix elements. A 4 by 4 matrix of correlators was computed using as basis states: local operator, fuzzed operator combined with the gamma matrix combinations $\gamma_{5}$ and $\gamma_{0} \gamma_{5}$. Two mass states were included in the fits. The heaviest state included in the fit is likely to have more excited state contamination in it.

The lattice axial current requires both matching factors to convert to $\overline{M S}$ scheme, as well as improvement factors. We use the formulation of the ALPHA collaboration 25].

$$
\left(A_{R}\right)_{\mu}^{a}=Z_{A}\left(1+b_{A} m_{q}\right)\left(A_{I}\right)_{\mu}^{a}
$$

We use the non-perturbative renormalisation and improvement factors recently computed by the ALPHA collaboration [26, 27] for two flavour unquenched QCD. At $\beta=5.2$ the ALPHA expressions give $c_{A}=-.0641$ [26]. At present, the $b_{A}$ factor is only known to one loop in perturbation theory.

The $f_{\pi^{\prime}}$ decay constant is extracted from the following combination.

$$
f_{\pi^{\prime}}=Z_{A}\left(1+b_{A} m\right)\left(a f_{A^{\prime}}+c_{A} f_{P^{\prime}}\right)
$$

where $f_{A^{\prime}}=\frac{1}{m^{\prime}}\left\langle 0\left|\bar{\psi} \gamma_{0} \gamma_{5} \psi\right| \pi^{\prime}\right\rangle$ and $f_{P^{\prime}}=\frac{1}{m^{\prime}}\left\langle 0\left|\partial_{4} \bar{\psi} \gamma_{5} \psi\right| \pi^{\prime}\right\rangle$.

In figure 2 we plot the ratio of the ratio of the decay constants of the $\pi$ and $\pi^{\prime}$ meson, using the unimproved and improved decay constants from the ALPHA formulation. On the $\mathrm{x}$-axis we use the square of the pion mass in 


\begin{tabular}{|c|c|c|}
\hline$\kappa$ & $\mathrm{a} f_{A^{\prime}}$ & $\mathrm{a} f_{P^{\prime}}$ \\
\hline 0.1358 & $0.042_{+6}^{-8}$ & $0.585_{-7}^{+4}$ \\
0.1355 & $0.065_{-8}^{+12}$ & $0.60_{-6}^{+6}$ \\
0.1350 & $0.11_{-2}^{+1}$ & $0.73_{-9}^{+6}$ \\
\hline
\end{tabular}

Table 2: Components of the $\pi^{\prime}$ decay constant. The ground state decay constants are in the second table of 21. The improved decay constant of the $\pi^{\prime}$ state is given by equation 7

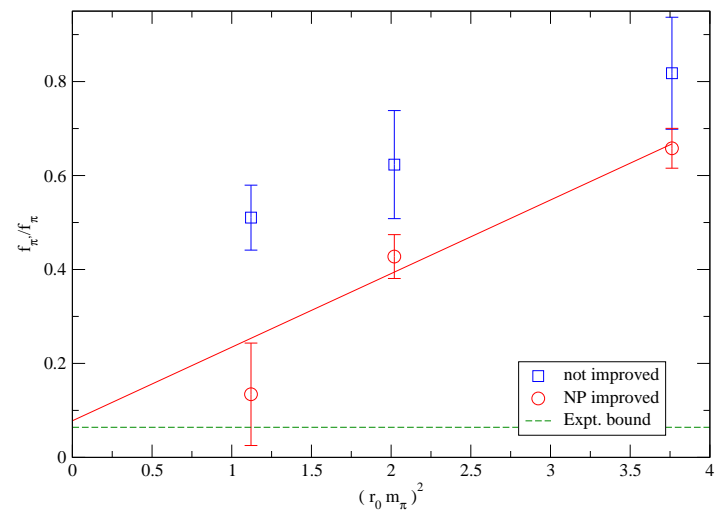

Figure 2: Ratio of the decay constants of the first excited to ground state light pseudo-scalar meson as a function of the pion mass squared. The horizontal line is the experimental upper limit from Diehl and Hiller [8].

units of $r_{0}$. The value of $r_{0}$ is not needed for our final result, but a value of $r_{0}$ around $0.5 \mathrm{fm}$ with $10 \%$ errors can be used if required [28. The unimproved decay constant has only a modest suppression of $f_{\pi^{\prime}}$ relative to $f_{\pi}$. The value of $f_{\pi^{\prime}}$ obtained from the improved ALPHA formulation is very much suppressed relative to $f_{\pi}$. In the continuum limit the improved and un-improved decay constants should agree. However, at the fixed lattice spacing that we use here, the improved decay constant should be close to the continuum limit value of the decay constant because the $O(a)$ errors have been removed.

A simple linear fit to the ratio of decay constants with improvement gives $f_{\pi^{\prime}} / f_{\pi}=0.078(93)$ in the chiral limit. The data at $\kappa=0.1358$ is important to in producing a smaller extrapolated ratio of decay constants. A simple linear extrapolation of the two heavier points gives $f_{\pi^{\prime}} / f_{\pi}=0.16$. For the unimproved decay constants, we obtain $f_{\pi^{\prime}} / f_{\pi}=0.38(11)$ in the chiral limit. The modest suppression of the $f_{\pi^{\prime}}$ decay constant when no improvement factors are used is 
consistent with the effective plots of the axial to pseudo-scalar channel looking similar to the pseudo-scalar to pseudo-scalar channel in figure 1 Although figure 2 could be viewed as a triumph of ALPHA's program of non-perturbative renormalisation, a controlled continuum extrapolation is required for a definitive result. Perhaps it is not so surprising that the full ALPHA formulation is required to see the suppression of the decay constant, because the PCAC relation is at the heart of the conditions imposed for improvement (determining coefficients of irrelevant operators) 25]. The increased suppression of the excited pion decay constant, from the improved current, is caused by the relatively large non-perturbative value of the coefficient of $c_{A}$ and the size of $f_{P^{\prime}}$. In quenched QCD at $\beta=6.0$, with a similar lattice spacing to the one used here, the value of $c_{A}$ was controversial with different methods giving different results [29, 30]. The ALPHA collaboration recommends that their formulation is used in a consistent continuum extrapolation ("working at constant physics") [26]. Also, there could be excited state contamination in the coupling $f_{P^{\prime}}$, that increases its value. To check the excited state contamination requires a larger variational basis.

In the original paper the UKQCD collaboration noted that the excited state masses were very close to the mass of three pions [21. It is not clear to us what we would expect for an axial current insertion into a three pion state. If we do a linear extrapolation of the masses of the $\pi^{\prime}$ meson versus the square of the pion mass in units of $r_{0}$ we obtain in the chiral limit: $m_{\pi^{\prime}}=1.29 \pm 0.29 \mathrm{MeV}$ (using a nominal value of $r_{0}=0.5 \mathrm{fm}$ ). Although the consistency of the extrapolated mass (within the large errors) with the mass of the $\pi(1300)$ state is good, it will be more interesting in future studies to monitor the effect of the three pion decay on the state.

\section{Conclusion}

We have investigated the leptonic decay constant of the first excited pseudoscalar meson. Although our results are consistent with $f_{\pi^{\prime}}$, being much smaller than $f_{\pi}$, a definitive lattice QCD calculation requires a continuum extrapolation. Recent algorithmic improvements have rescued Wilson/clover lattice calculations from being too computationally expensive with light quark masses, hence it is now possible to repeat this calculation with more than one lattice spacing and lighter quarks [31, 32, 33.

The suppression of the excited light pseudo-scalars may have implications for the proposed restoration of chiral symmetry for highly excited hadrons, suggested by Glozman 34. If the leptonic decay constant of the excited pseudoscalar mesons are suppressed, then the leptonic decay constant of the parity partners of the pseudo-scalar mesons should also be suppressed. Although, unfortunately, lattice QCD is not ideally suited to studying highly excited mesons, the study of the suppression of the decay constant of the first excited light pseudo-scalar is an interesting first step. Note that even if lattice QCD cannot be used to study the proposed restoration of chiral symmetry in the excited hadron spectrum, it may be able to study the proposed "restoration of chiral 
symmetry" via the eigenvalue spectrum [35, 36].

Following Narison [37, in [38 we argued that for the light $0^{++}$state a small value for the leptonic decay constant would be a signal for a molecular state. This seems an unlikely interpretation for the smallness of the decay constant of the $\pi^{\prime}$ meson [14.

The suppression of $f_{\pi^{\prime}}$ is a useful "benchmark" that can be used to tune and validate lattice QCD techniques that try to determine the properties of excited states mesons. It is particularly interesting, because it is a consequence of chiral symmetry. Holl et al. [39] also have predictions for the sign of the decay constant of the $\pi^{\prime}$ meson that may also be studied using lattice QCD.

\section{Acknowledgements}

We thank Craig Roberts for bringing this issue to our attention. We thank Alexander Andrianov for comments. This work has been supported in part by the EU Integrated Infrastructure Initiative Hadron Physics (I3HP) under

contract RII3-CT-2004-506078. We are grateful to the ULgrid project of the University of Liverpool for computer time. One of the authors (CM) wishes to thank PPARC for the award of a Senior Fellowship.

\section{References}

[1] A. Holl, A. Krassnigg, and C. D. Roberts, Phys. Rev. C70, 042203 (2004), nucl-th/0406030

[2] C. A. Dominguez and M. Moreno, Phys. Rev. D16, 856 (1977),

[3] A. A. Andrianov, V. A. Andrianov, and A. N. Manashov, Int. J. Mod. Phys. A6, 5435 (1991),

[4] M. K. Volkov and C. Weiss, Phys. Rev. D56, 221 (1997), hep-ph/9608347,

[5] V. Elias, A. Fariborz, M. A. Samuel, F. Shi, and T. G. Steele, Phys. Lett. B412, 131 (1997), hep-ph/9706472.

[6] A. A. Andrianov, D. Espriu, and R. Tarrach, Nucl. Phys. B533, 429 (1998), hep-ph/9803232

[7] K. Maltman and J. Kambor, Phys. Rev. D65, 074013 (2002), hep-ph/0108227.

[8] M. Diehl and G. Hiller, JHEP 06, 067 (2001), hep-ph/0105194,

[9] W. Lucha, D. Melikhov, and S. Simula, (2006), hep-ph/0606281

[10] O. Lakhina and E. S. Swanson, (2006), hep-ph/0603164 
[11] J. J. Dudek, R. G. Edwards, and D. G. Richards, Phys. Rev. D73, 074507 (2006), hep-ph/0601137.

[12] C. Michael, Phys. Rev. 166, 1826 (1968).

[13] Particle Data Group, S. Eidelman et al., Phys. Lett. B592, 1 (2004),

[14] T. Barnes, F. E. Close, P. R. Page, and E. S. Swanson, Phys. Rev. D55, 4157 (1997), hep-ph/9609339,

[15] ClEO, D. M. Asner et al., Phys. Rev. D61, 012002 (2000), hep-ex/9902022

[16] T. Burch et al., Phys. Rev. D73, 094505 (2006), hep-lat/0601026,

[17] CP-PACS, T. Yamazaki et al., Phys. Rev. D65, 014501 (2002), hep-lat/0105030

[18] C. Aubin et al., Phys. Rev. D70, 094505 (2004), hep-lat/0402030,

[19] N. V. Krasnikov and A. A. Pivovarov, Phys. Lett. B112, 397 (1982),

[20] A. L. Kataev, N. V. Krasnikov, and A. A. Pivovarov, Phys. Lett. B123, 93 (1983),

[21] UKQCD, C. R. Allton et al., (2004), hep-lat/0403007.

[22] C. McNeile, (2003), hep-lat/0307027.

[23] UKQCD, C. R. Allton et al., Phys. Rev. D65, 054502 (2002), hep-lat/0107021

[24] JLQCD, S. Aoki et al., Phys. Rev. D68, 054502 (2003), hep-lat/0212039,

[25] K. Jansen et al., Phys. Lett. B372, 275 (1996), hep-lat/9512009,

[26] M. Della Morte, R. Hoffmann, and R. Sommer, JHEP 03, 029 (2005), hep-lat/0503003

[27] ALPHA, M. Della Morte et al., Nucl. Phys. B729, 117 (2005), hep-lat/0507035

[28] R. Sommer, Nucl. Phys. B411, 839 (1994), hep-lat/9310022

[29] T. Bhattacharya, R. Gupta, W.-J. Lee, and S. R. Sharpe, Phys. Rev. D63, 074505 (2001), hep-lat/0009038

[30] UKQCD, S. Collins, C. T. H. Davies, G. P. Lepage, and J. Shigemitsu, Phys. Rev. D67, 014504 (2003), hep-lat/0110159

[31] M. Hasenbusch, Phys. Lett. B519, 177 (2001), hep-lat/0107019,

[32] C. Urbach, K. Jansen, A. Shindler, and U. Wenger, Comput. Phys. Commun. 174, 87 (2006), hep-lat/0506011. 
[33] M. Luscher, PoS LAT2005, 002 (2006), hep-lat/0509152.

[34] L. Y. Glozman, Phys. Lett. B587, 69 (2004), hep-ph/0312354.

[35] T. A. DeGrand, Phys. Rev. D69, 074024 (2004), hep-ph/0310303,

[36] T. D. Cohen, (2006), hep-ph/0605206.

[37] S. Narison, (2005), hep-ph/0512256

[38] UKQCD, C. McNeile and C. Michael, (2006), hep-lat/0604009.

[39] A. Holl, A. Krassnigg, P. Maris, C. D. Roberts, and S. V. Wright, Phys. Rev. C71, 065204 (2005), nucl-th/0503043. 